\title{
Crack Propagation Analysis in Laser Scribing of Glass
}

\author{
Keisuke YAHATA $^{* 1}$, Koji YAMAMOTO*1 and Etsuji OHMURA ${ }^{* 2}$ \\ ${ }^{*}$ Mitsuboshi Diamond Industrial Co.,LTD., 1-4-37 Minamikaneden, Suita, Osaka 564-0044, Japan \\ E-mail: kyahata@mitsuboshi-dia.co.jp \\ ${ }^{* 2}$ Division of Materials and Manufacturing Science, Osaka University, 2-1 Yamada-oka, Suita, \\ Osaka 565-0871, Japan
}

\begin{abstract}
In the laser scribing of a glass sheet, a crack propagates across the glass surface as the surface is locally cooled down immediately following $\mathrm{CO}_{2}$ laser beam irradiation. In this process, the crack depth and scribable conditions are influenced by various parameters such as the laser power, the scribing velocity, and the distance between the heating area and the cooling area. Therefore it is important to examine the laser scribing phenomenon by analyses of these parameters. We analyzed the crack propagation phenomenon by a finite element method, using a two-dimensional model with a pre-crack. The crack depth was obtained by calculating the stress intensity factor. The obtained conclusions are as follows: The maximum velocity of the scribing for each laser power can be estimated by applying a static threshold of the stress intensity factor, $K_{\text {sth }}$ in the two-dimensional crack propagation analysis. The depth of the crack caused by the laser scribing can be estimated by applying a dynamic threshold of the stress intensity factor, $K_{\text {Dth }}$. As a result, the suitable scribing conditions for the practical use can be found.
\end{abstract}

DOI:10.2961/jlmn.2010.02.0002

Keywords: laser scribing, glass, $\mathrm{CO}_{2}$ laser, thermal stress analysis, crack

\section{Introduction}

In recent years, flat panel displays, laptop computers, and cell phones have been spreading fast, and the demands of liquid crystal displays (LCD) and plasma display panels (PDP) used in these products are growing. In the manufacturing of LCDs or PDPs, the glass cutting process is indispensable component.

Currently, glass is mainly cut by inducing a bending stress after scribing the glass with a cutter wheel. In this mechanical process, particles are inevitably generated [1], and further, microcracks generated on the glass surface decrease the strength of the glass. Especially for portable products, a high bending strength is demanded and therefore, fewer microcracks should be generated.

In 1993, the laser scribing process was developed [2] in which a crack grows as glass is cooled down immediately after laser beam irradiation. Fig. 1 shows the laser scribing mechanism [3]. When the glass surface is cooled down by the water jet, a tensile stress is generated under the surface. This time the inside still has a high temperature caused by the laser beam irradiation and a compressive stress is generated. This compressive stress increases the tensile stress, and the crack grows. It is reported that fewer particles are

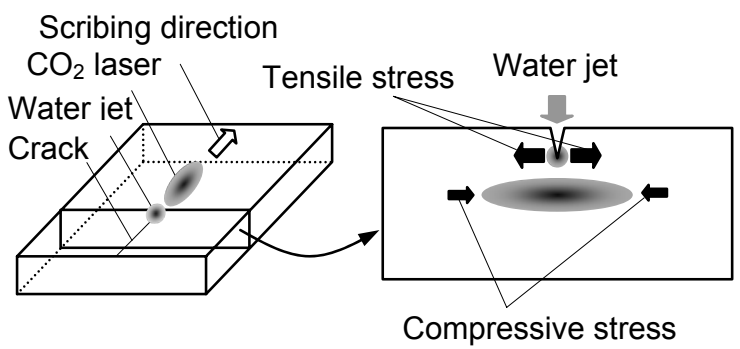

Fig. 1 Principle of laser scribing generated and that the bending strength of the broken glass is increased by this process [4].

Due to these advantages, the laser scribing process is suitable for cutting of glass substrates, therefore, the processing mechanism has been examined by analytical and experimental studies [3, 5]. However neither an analysis based on fracture mechanics nor research into crack depth estimation has been carried out. Accordingly in this research, we aim to estimate the crack depth achieved in the laser scribing of a glass sheet and to obtain the most suitable condition for laser scribing. We conducted twodimensional thermal elastic analyses based on fracture mechanics. In the analyses, we use a model with a pre-crack, and the crack propagation phenomenon is considered by the stress intensity factor.

\section{Laser scribing experiment}

\subsection{Measurement of crack depth}

We conduct a laser scribing experiment for soda lime

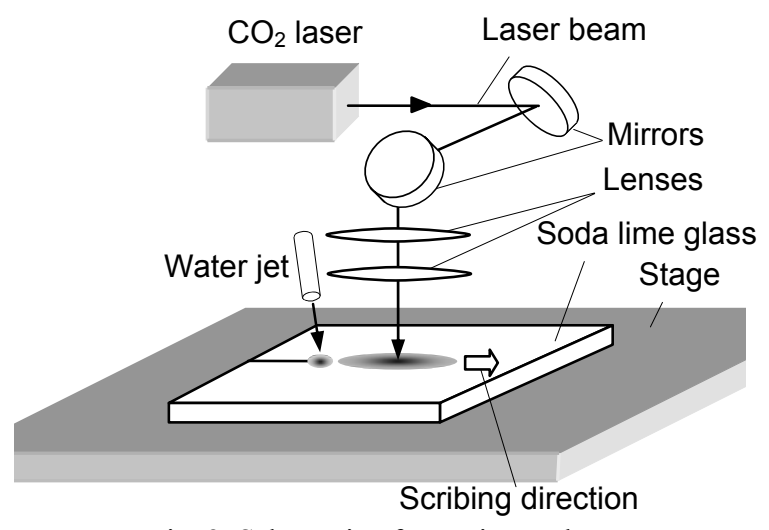

Fig. 2 Schematic of experimental setup 
glass having dimensions of $230 \mathrm{~mm} \times 360 \mathrm{~mm}$, and a thickness of $700 \mu \mathrm{m}$, in order to measure the depth of the crack caused by the laser scribing. Fig. 2 shows the schematic of the experimental set up. After the glass is fixed on the stage by vacuum absorption, an initial crack is formed by a cutter wheel at the starting point of the scribing. The laser beam is shaped into an ellipsoid by two lenses and is then irradiated onto the glass surface. At the same time, an area behind the laser beam along the extended line of the major axis of the heating area is cooled down by the water jet. The distance between the center of the heating area and the center of the cooling area is called cooling point distance, and is written as $d$ afterward. The laser beam and the water jet are scanned at a constant velocity, $v$ by moving the stage in the direction from the laser beam to the water jet. Table 1 shows the experimental conditions.

After the scribing, the glass is broken along the scribed line. Depth of the crack caused by the laser scribing is measured by observing the cutting plane. Fig. 3 shows the measured crack depth, $D_{\mathrm{c}}$ for the conditions of the cooling point distance, $d 10 \mathrm{~mm}$, the laser power, $P$ and the scribing velocity, $v$ variable. For each value of $P$, when $v$ is decreased, thermal damage is caused on the glass surface. These conditions are not useful due to a decrease of strength. When $v$ is increased, the crack propagation stops along the direction of scribing. These conditions, where thermal damage occurs or crack propagation is halted are judged unscribable. In this research, conditions producing crack propagation with no thermal damage are called the scribable conditions. Fig. 3 shows $D_{\mathrm{c}}$ under the scribable conditions.

\subsection{Time variation of crack propagation}

In order to investigate how the crack grows in laser scribing, the crack development is stopped along the direction of scribing by interruption of the water jet impingement. After that, the glass is broken along the scribed line, and the shape of the crack end in the cutting plane is observed. Fig. 4 shows one example of the light microscope images. In the laser scribing, a crack is considered to develop in the scribing direction with a knife shape as shown in Fig. 4.

\section{Analysis method}

Found from Fig. 4, in the laser scribing, the crack propagates in the thickness direction as it develops in the scribing direction. Therefore three-dimensional analyses can examine the crack propagation phenomenon closer to the real one. However three-dimensional analyses take too much time. Giving priority consideration to the practicality, we conducted two-dimensional analyses in this research.

\subsection{Thermal analysis}

The direction of the minor axis of the heating area is defined to be the $x$-direction, the scribing direction to be the $y$-direction, and the thickness direction to be the $z$-direction. We conducted two-dimensional thermal analyses for one $x-z$ cross section by a finite element method. Fig. 5 shows the mesh geometry for thermal analyses. Because of the symmetry of the laser scribing phenomenon, a half model is used. In the analysis plane, the area near the symmetric line is divided smaller due to the violent varia-
Table. 1 Experimental conditions

\begin{tabular}{cl|l}
\hline$v$ & Scribing velocity & $40 \mathrm{~mm} / \mathrm{s} \sim 400 \mathrm{~mm} / \mathrm{s}$ \\
$P$ & Laser power & $30.4 \mathrm{~W} \sim 80.5 \mathrm{~W}$ \\
$2 x_{\mathrm{h}}$ & Minor axis of heating area & $2.1 \mathrm{~mm}$ \\
$2 y_{\mathrm{h}}$ & Major axis of heating area & $22.0 \mathrm{~mm}$ \\
$d$ & Cooling point distance & $8 \mathrm{~mm} \sim 26 \mathrm{~mm}$ \\
$2 x_{\mathrm{c}}$ & Minor axis of cooling area & $2.0 \mathrm{~mm}$ \\
$2 y_{\mathrm{c}}$ & Major axis of cooling area & $3.0 \mathrm{~mm}$ \\
\hline
\end{tabular}

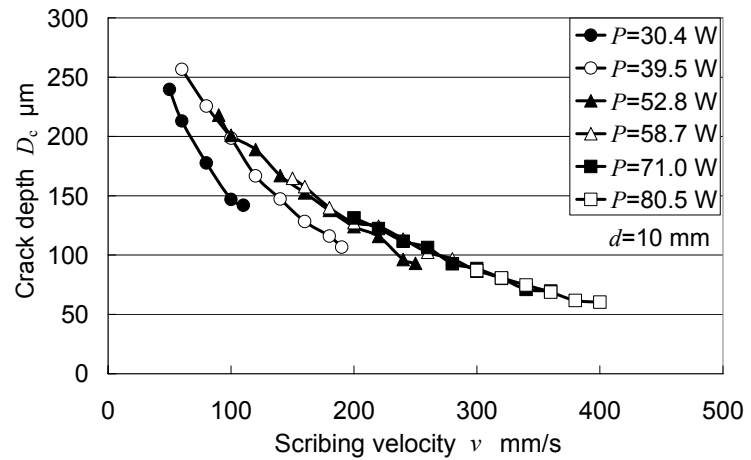

Fig. 3 Measured crack depth under scribable conditions

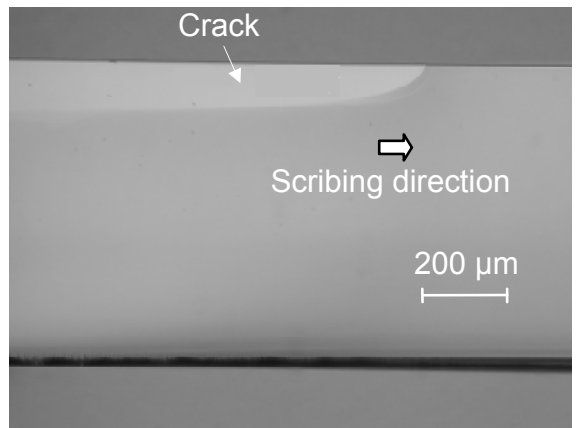

Fig. 4 Picture of cutting plane when crack propagation is stopped

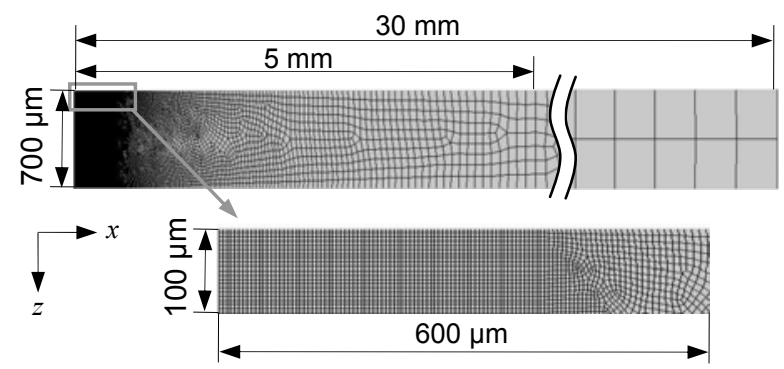

Fig. 5 Mesh geometry for thermal analysis

tion of the temperature. The smallest element is a square one $5 \mu \mathrm{m} \times 5 \mu \mathrm{m}$ in dimensions. The number of nodes is 18716 , and the number of elements is 18324 .

Figure 6 shows the heating area and the cooling area in thermal analyses. The heating by the laser beam is modeled by applying the elliptic Gaussian heat flux, $q$ on the upper side of the model and by moving $q$ in the $y$-direction at a velocity of $v$. The values of $x_{\mathrm{h}}$ and $y_{\mathrm{h}}$ shown in table 1 are used as the $1 / e^{2}$ radii of $q$ respectively in the $x$ and $y$ directions, and $q$ is defined by equation (1). $0.80 P$ is 
substituted for the amount of heat input $P^{\prime}$, considering the damping ratio of the optical system and the reflectance of soda lime glass.

$$
q=\frac{2 P^{\prime}}{\pi x_{\mathrm{h}} y_{\mathrm{h}}} \exp \left[-2\left(\frac{x^{2}}{x_{\mathrm{h}}{ }^{2}}+\frac{y^{2}}{y_{\mathrm{h}}{ }^{2}}\right)\right]
$$

The cooling by the water jet is modeled by applying the elliptic Gaussian heat transfer coefficient, $\alpha$ in the area $d$ apart from the center of the heating area. $\alpha$ also moves in the $y$-direction at a velocity of $v$. The values of $x_{\mathrm{c}}$ and $y_{\mathrm{c}}$ shown in table 1 are used for the $1 / e^{2}$ radii of the cooling area respectively in the $x$ and $y$ directions, and $\alpha$ is defined by equation (2). The heat transfer coefficient at the collision point $\alpha_{\max }$ was calculated [6] from the flow rate of the water jet. In this experiment, the flow rate of the water jet was $0.8 \mathrm{ml} / \mathrm{min}$, and then, $\alpha_{\max }$ was calculated to be $1.0 \times 10^{5} \mathrm{~W} / \mathrm{m}^{2} \mathrm{~K}[7]$.

$$
\alpha=\alpha_{\max } \exp \left[-2\left(\frac{x^{2}}{x_{\mathrm{c}}{ }^{2}}+\frac{y^{2}}{y_{\mathrm{c}}{ }^{2}}\right)\right]
$$

The time step is the needed time for the laser beam or the water jet to proceed $125 \mu \mathrm{m}$. All boundaries of the analysis model are defined to be insulated. Initial temperature is set to be $20^{\circ} \mathrm{C}$. The physical properties shown in table 2 [8-10] are applied, and time variation of temperature distribution is analyzed.

\subsection{Thermal stress analysis}

Based on results of the thermal analyses, we conduct thermal stress analyses as a plane strain problem. Shown in Fig. 7 , in thermal stress analyses, a pre-crack at a depth of $d_{\mathrm{p}}$ is set on the upper side of the model, and isoparametric elements $[11,12]$ are placed around the crack tip. Their intermediate nodes are not on the middle of their sides, and they can reflect the singular stress field around the crack tip in results. On the symmetric line $(x=0)$, the nodes on the crack part are bound to have the positive $x$-displacement, $u_{x}$, and the others not to have $u_{x}$. The crack's opening is modeled by this nodal binding. And on $x=30 \mathrm{~mm}$, the nodal displacement in all directions are bound to be 0 . The number of nodes is 60296 , and the number of elements is 19752.

\subsubsection{Thermal stress analysis in which crack depth is constant (static analysis)}

By the above model, time variation of the stress intensity factor, $K_{\mathrm{I}}$ is obtained with the crack depth constant. $K_{\mathrm{I}}$ is calculated by equation (3) [13], using the nodal $x$ displacement, $u_{x}$ on the crack part. $G$ is the modulus of transverse elasticity, $v$ is the Poisson's ratio, and $r$ is the distance from the crack tip.

$$
K_{\mathrm{I}}=\lim _{r \rightarrow 0} \frac{G u_{x}}{2(1-v)} \sqrt{\frac{2 \pi}{r}}
$$

In this research, this thermal stress analysis in which the crack depth is constant is called a static analysis.

\subsubsection{Crack propagation analysis}

In order to obtain the crack depth in the laser scribing by an analysis, we conduct another thermal stress analysis

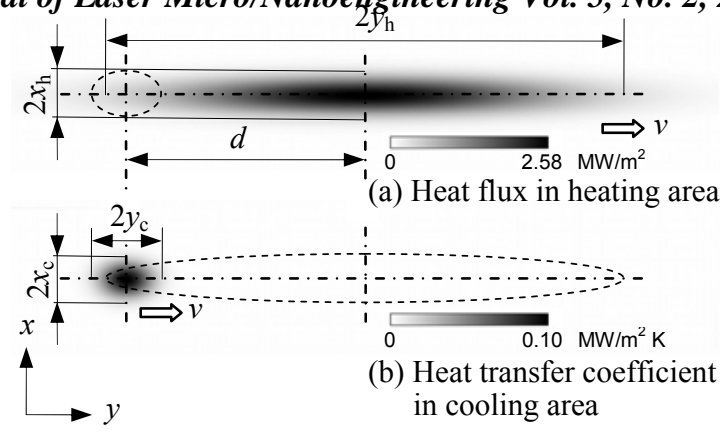

Fig. 6 Heating area and cooling area in thermal analysis

Table. 2 Physical properties of soda lime glass

\begin{tabular}{l|l}
\hline Density & $2520 \mathrm{~kg} / \mathrm{m}^{3}$ \\
Specific heat & $800 \mathrm{~J} / \mathrm{kg} \mathrm{K}$ \\
Thermal conductivity & $1.03 \mathrm{~W} / \mathrm{m} \mathrm{K}$ \\
Thermal expansion coefficient & $8.7 \times 10^{-6} \mathrm{~K}^{-1}$ \\
Young's modulus & $71.6 \mathrm{GPa}$ \\
Poisson's ratio & 0.23 \\
\hline
\end{tabular}

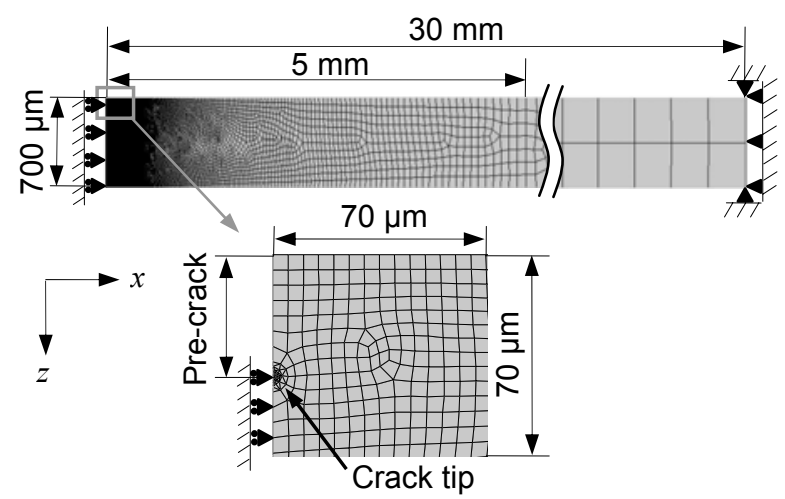

Fig. 7 Mesh geometry for thermal stress analysis

in which the crack propagates. This analysis also starts with a pre-crack at a depth of $d_{\mathrm{p}}$, and the stress intensity factor, $K_{\mathrm{I}}$ is calculated at every time step. Applying thresholds of the stress intensity factor, the crack propagates $\Delta d$ in the thickness direction every time $K_{\mathrm{I}}$ exceeds the threshold.

As mentioned above, in laser scribing, the crack propagates in the thickness direction as it develops in the scribing direction. Therefore in this two-dimensional analysis, a different threshold is applied respectively for the first propagation in the analysis plane and the other propagation. One threshold for the first propagation is called a static threshold and written as $K_{\text {Sth }}$. The other is called a dynamic threshold and written as $K_{\text {Dth }}$. In other words, crack propagates $\Delta d$ for the first time when $K_{\mathrm{I}}$ exceeds $K_{\text {Sth }}$. Then the threshold is changed, and the crack propagates $\Delta d$ by $\Delta d$ every time $K_{\mathrm{I}}$ exceeds $K_{\text {Dth }}$.

When the crack propagates, the model is remeshed, and the isoparametric elements are placed around the new crack tip deeper by $\Delta d$. And the bounding conditions are reset as the following. On $x=0, u_{x} \geq 0$ for nodes on the crack part, and $u_{x}=0$ for the other nodes. Besides, the crack is allowed to propagate several times at one time step, because $K_{\mathrm{I}}$ is calculated again under the same temperature distribution after the crack propagates. 
The crack depth during the analysis is written as $\tilde{d}_{\mathrm{c}} . \tilde{d}_{\mathrm{c}}$ at the end of the analysis is defined to be the analyzed crack depth in the laser scribing, $\widetilde{D}_{\mathrm{c}}$. If $K_{\mathrm{I}}$ doesn't exceed

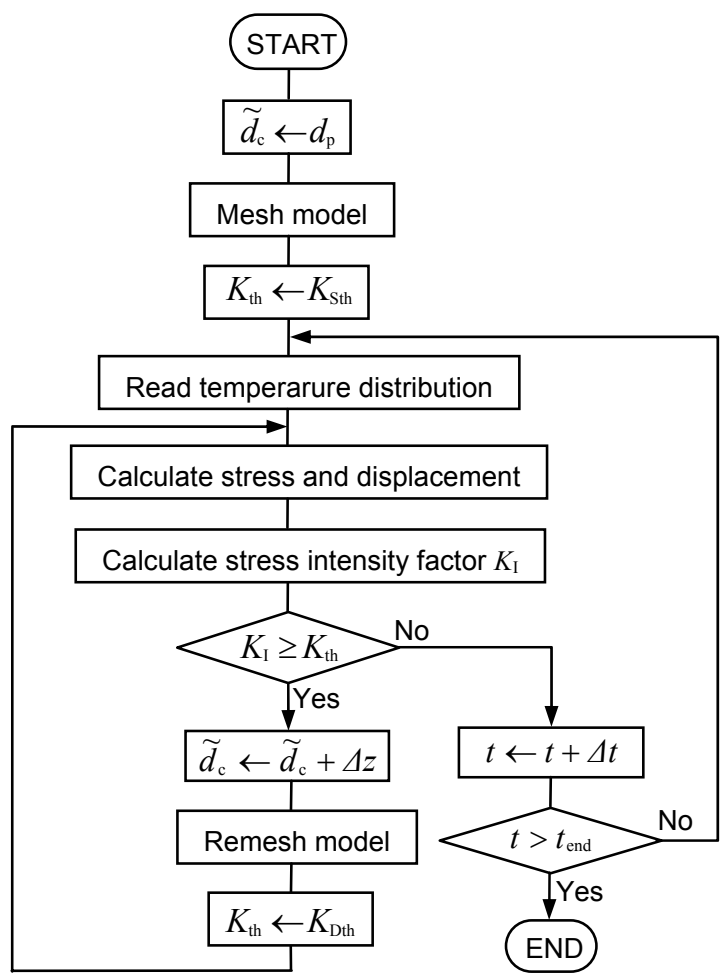

Fig. 8 Flowchart of crack propagation analysis

\subsection{Results of thermal analysis}

Figure 9 shows one example of results of the thermal analysis in the conditions of $P=58.7 \mathrm{~W}, v=200 \mathrm{~mm} / \mathrm{s}$, and $d=10 \mathrm{~mm}$. Immediately before the cooling area gets to the analysis plane, the surface temperature reaches the maximum value, $T_{\max }$. After that the glass surface is cooled down when the cooling area passes across the analysis plane, and the heated area exists inside the model.

When the laser power, $P$ is constant, $T_{\max }$ is increased with the decrease of the scribing velocity, $v$. In six minimum velocity conditions with no thermal damage in each $P$ of Fig. 3, the mean value of $T_{\max }$ is $496^{\circ} \mathrm{C}$. It is reported that thermal damage is generated on the surface of soda lime glass when $T_{\max }$ exceeds about $500^{\circ} \mathrm{C}$ [3]. Therefore also in this analysis, the upper limit of $T_{\max }$ with no thermal damage is about $500^{\circ} \mathrm{C}$. Also, the scribable minimum velocity can be estimated by $T_{\max }$.

\subsection{Results and consideration of thermal stress analyses 4.2.1 Determination of pre-crack depth}

We conduct static analyses, applying a variety of pre-crack depths, $d_{\mathrm{p}}$. Fig. 10 shows an example of the variations in the stress intensity factor, $K_{\mathrm{I}}$ when the cooling area crosses the analysis plane in the conditions of $P=58.7 \mathrm{~W}, v=200 \mathrm{~mm} / \mathrm{s}$, and $d=10 \mathrm{~mm}$. The horizontal axis means the location of the center of the cooling area for the analysis plane. $y<0$ means that the center of the cooling area is behind the analysis plane, and $y>0$ means it is in front of the analysis plane. $K_{\mathrm{I}}$ is rapidly increased as the

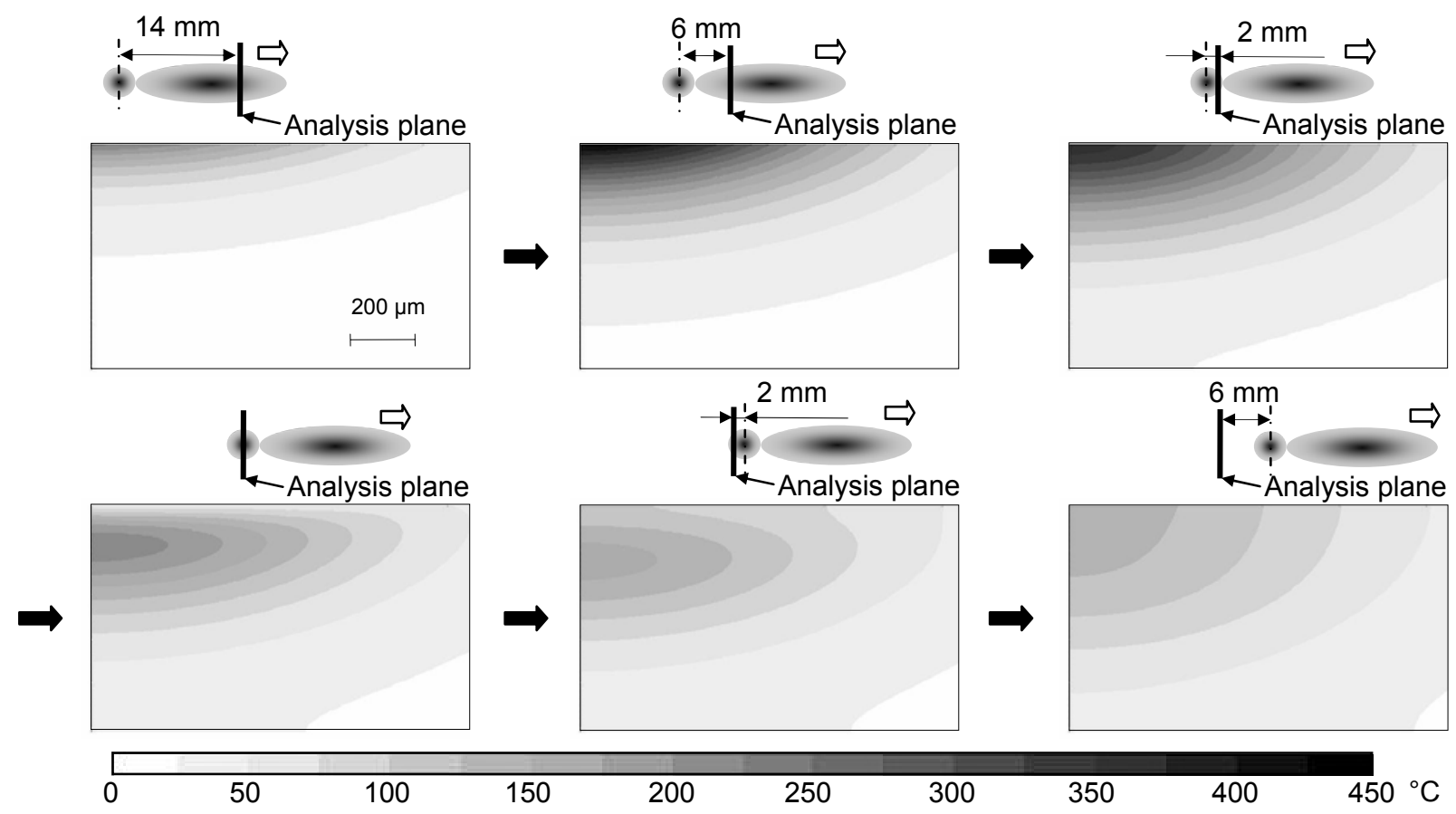

Fig. 9 Result of thermal analysis in conditions of $P=58.7 \mathrm{~W}, v=200 \mathrm{~mm} / \mathrm{s}, d=10 \mathrm{~mm}$

$K_{\text {sth }}, \widetilde{D}_{\text {c }}$ is defined to be 0 . In this research, this analysis is called a crack propagation analysis whose flowchart is shown in Fig. 8. Here $\Delta d$ is set to be $5 \mu \mathrm{m}$. cooling area approaches the analysis plane, and there exists the maximum value which is written as $K_{\max }$. It is found that $K_{\max }$ depends on the pre-crack depth, $d_{\mathrm{p}}$.

\section{Analysis results and consideration}


Because the crack development stops at the maximum velocity of the scribing, $K_{\max }$ must be constant in six conditions at the maximum velocity in each laser power, $P$ of Fig. 3. And the value of $K_{\max }$ must be equal to the threshold of crack development. Then $K_{\max }$ is obtained in these six conditions, applying a variety of $d_{\mathrm{p}}$. Fig. 11 shows the results. If $d_{\mathrm{p}} \geq 20 \mu \mathrm{m}$, there exists large variability in $K_{\max }$ as $d_{\mathrm{p}}$ is increased. On the other hand if $d_{\mathrm{p}} \leq 15 \mu \mathrm{m}$, then less variability exists, and the standard deviation of $K_{\max }$ is the minimum when $d_{\mathrm{p}}$ is $10 \mu \mathrm{m}$. For these reasons $d_{\mathrm{p}}$ is determined to be $10 \mu \mathrm{m}$.

\subsubsection{Determination of static threshold}

The crack development in the laser scribing phenomenon is equal to the start of the crack propagation in the crack propagation analysis. Therefore the static threshold, $K_{\text {Sth }}$ is equal to the mentioned threshold of the crack development. Then $K_{\text {Sth }}$ should be determined from the range of $K_{\max }$ of Fig. 11 in the condition of $d_{\mathrm{p}} 10 \mu \mathrm{m}$. By applying the maximum value of the $K_{\max }$ range for $K_{\text {sth }}$, estimation of too wide range of the scribable velocity is prone to be avoided. From these reasons, the static threshold, $K_{\text {sth }}$ is determined to be $0.50 \mathrm{MPa} \mathrm{m}{ }^{1 / 2}$.

\subsubsection{Determination of dynamic threshold}

In order to determine the dynamic threshold, $K_{\text {Dth }}$, the analyzed crack depth, $\widetilde{D}_{\mathrm{c}}$ is obtained by the crack propagation analysis, applying a variety of $K_{\text {Dth }}$. Fig. 12 shows the results in three scribing conditions. The measured crack depth $D_{\mathrm{c}}$ in each condition is shown by a broken line. In each condition, $\widetilde{D}_{\mathrm{c}}$ is decreased with the increase of $K_{\mathrm{Dth}}$. So $\widetilde{D}_{\mathrm{c}}$ approaches $D_{\mathrm{c}}$ by applying suitable $K_{\mathrm{Dth}}$. However $K_{\text {Dth }}$ does not exist which minimizes the errors of the crack depth in all conditions.

Then $K_{\mathrm{Dth}}$ is obtained which makes the relative errors of $\widetilde{D}_{\mathrm{c}}$ for $D_{\mathrm{c}}$ comparatively small in all conditions by the following method. First eighteen scribable conditions are selected three by three from each laser power, $P$ of Fig. 3. $\widetilde{D}_{\mathrm{c}}$ is obtained in these eighteen conditions, applying a variety of $K_{\text {Dth }}$, and errors are calculated by equation (4).

$$
\varepsilon=\frac{\left|\widetilde{D}_{\mathrm{c}}-D_{\mathrm{c}}\right|}{D_{\mathrm{c}}} \times 100
$$

Figure 13 shows the mean value of eighteen errors, $\bar{\varepsilon}$. The standard deviation of $\varepsilon$ is shown by the error bars. $\bar{\varepsilon}$ is minimized when $K_{\text {Dth }}$ is $0.37 \mathrm{MPa} \mathrm{m}^{1 / 2}$. And the dynamic threshold, $K_{\text {Dth }}$ is determined to be $0.37 \mathrm{MPa} \mathrm{m}^{1 / 2}$.

\subsubsection{Availability of two thresholds of stress intensity factor}

Figure 14 shows the analyzed and measured crack depth, $\widetilde{D}_{\mathrm{c}}$ and $D_{\mathrm{c}}$ in the conditions of $d=10 \mathrm{~mm}$ and $P=39.5 \mathrm{~W}, 52.8 \mathrm{~W}, 71.0 \mathrm{~W}$. $\widetilde{D}_{\mathrm{c}}$ accords well with $D_{\mathrm{c}}$ in almost all conditions by setting $K_{\text {Dh }}$ to $0.37 \mathrm{MPa} \mathrm{m}^{1 / 2}$. And the analyzed maximum velocity of the scribing also accords well with the experimental results by setting $K_{\text {Sth }}$ to $0.50 \mathrm{MPa} \mathrm{m}{ }^{1 / 2}$ which is the threshold of the crack development.

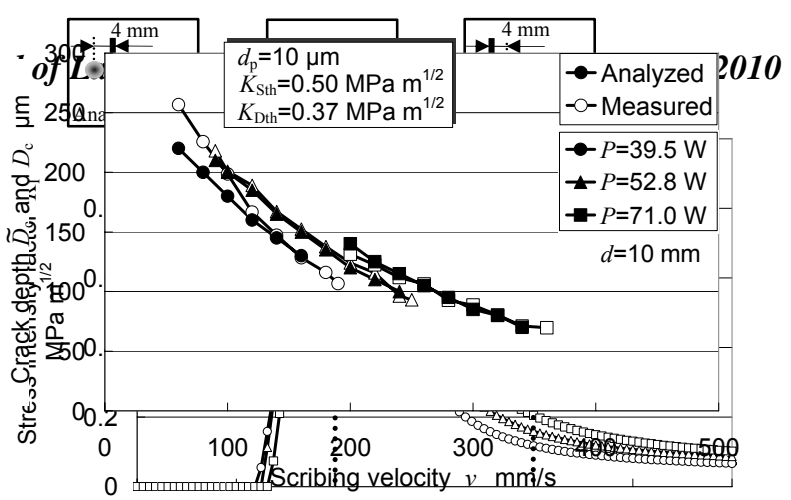

Fig. 14 Gompari\&on of measure $\$$ and analyzed crack 8

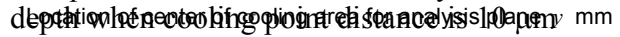

Fig. 10 Stress intensity factor versus location of center of cooling area when pre-crack depth is varied

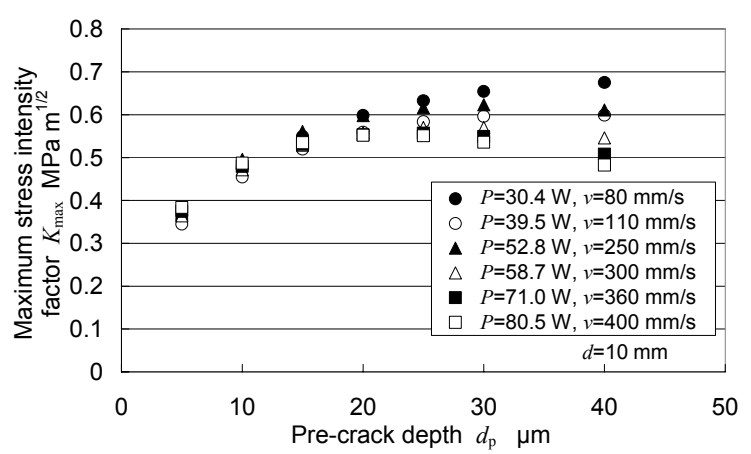

Fig. $11 K_{\max }$ at scribable maximum velocity when pre-crack depth is varied

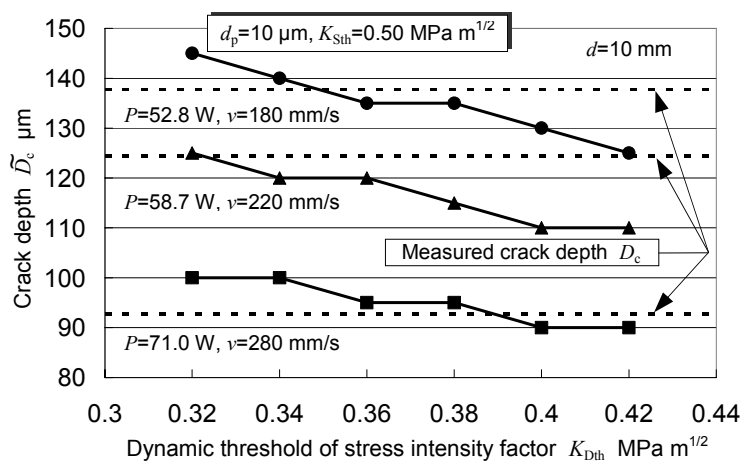

Fig. 12 Analyzed crack depth versus dynamic threshold of stress intensity factor

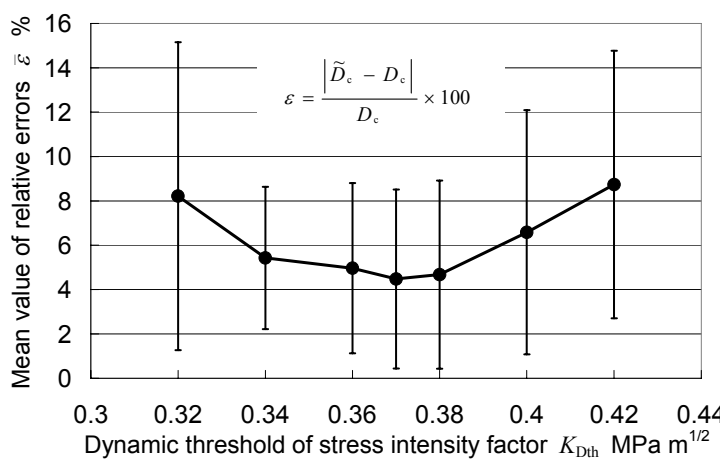

Fig. 13 Mean value of $\varepsilon$ versus dynamic threshold of stress intensity factor

Figure 15 shows $\widetilde{D}_{\mathrm{c}}$ and $D_{\mathrm{c}}$ in the scribable conditions with $P$ and $v$ constant and the cooling point distance, $d$ variable. $\widetilde{D}_{\mathrm{c}}$ is obtained in the conditions of $K_{\text {sth }} 0.50$ $\mathrm{MPa} \mathrm{m}^{1 / 2}$ and $K_{\mathrm{Dth}} 0.37 \mathrm{MPa} \mathrm{m} \mathrm{m}^{1 / 2}$. The analyzed crack 


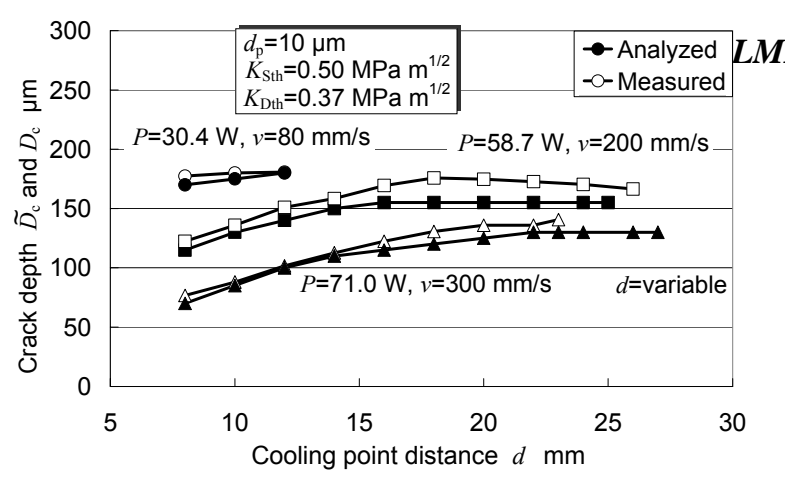

Fig. 15 Comparison of measured and analyzed crack depth when cooling point distance is varied

depth accords well with the experimental results even when $d$ is varied. From these results, it is found that $K_{\mathrm{Dht}}$ determined by using the conditions of $d 10 \mathrm{~mm}$ can be used even when $d$ is not $10 \mathrm{~mm}$.

In Fig. 15, the analyzed maximum value of the scribable cooling point distance accords comparatively well with the experimental results. In the experiment, when the cooling point distance, $d$ is increased with $P$ and $v$ constant, the condition shifts to unscribable due to interruption of crack development. From these results, $K_{\text {sth }}$ determined by using conditions of $d 10 \mathrm{~mm}$ can be used even when $d$ is not $10 \mathrm{~mm}$ as a threshold of the crack development.

From the above, the maximum scribing velocity can be estimated by the static threshold, $K_{\text {sth }}$ and the crack depth can be by the dynamic threshold, $K_{\mathrm{Dth}}$. As mentioned above, the scribable minimum velocity can be estimated by the maximum temperature of the glass surface, $T_{\max }$. Therefore the range of the scribable velocity can be estimated by this research for a variety of laser power, $P$. As a result, in the practical use, the stable scribing quality can be obtained by selecting the velocity around the middle of the scribable range. And because the crack depth can be estimated in each condition, the suitable conditions can be further narrowed down.

\section{Conclusions}

In this research, we aim to estimate the crack depth in the laser scribing of a glass sheet and to obtain the most suitable scribable condition easily. And we conduct two-dimensional thermal elastic analyses based on fracture mechanics. In the analyses, the crack propagation phenomenon is considered, applying the static and dynamic

thresholds of the stress intensity factor, $K_{\text {Sth }}$ and $K_{\text {Dth }}$. The obtained conclusions are as follows:

1. The scribable maximum velocity can be estimated by the static threshold, $K_{\text {Sth }}$. And the scrabable minimum velocity can be estimated by the maximum temperature, $T_{\max }$.

2. The crack depth in the laser scribing can be estimated by the dynamic threshold, $K_{\text {Dth }}$.

3. In the practical use, the stable scribing quality can be obtained by selecting the velocity around the middle of the analyzed scribable velocity range.

4. Because the crack depth can be estimated in each condition, the suitable conditions can be further narrowed down.

\section{References}

[1] S.C. Wang, L.Y. Yeh, C.C. Lin, M.S. Chen and F.Y. Gan: Proc. IDW'07, (2007) p.513. (Conference Proceedings)

[2] V.S. Kondratenko: PTC WO 20015 (1993) (Patent)

[3] K. Yamamoto, N. Hasaka, H. Morita and E. Ohmura: J. Laser Appl., 20 (2008) 193. (Journals)

[4] Y. Miyake: J. Jpn. Soc. Abras. Technol, 45 (2001) 342. (in Japanese)

[5] C. Tsai, B. Lin: Int. J. Adv. Manuf. Technol., 32 (2007) 1155. (Journals)

[6] A. Yamamoto: J. Jpn. Soc. Prec. Eng., 26 (1960) 461. (in Japanese)

[7] K. Yamamoto, H. Morita, N. Hasaka, E. Ohmura, Pre. Eng., 34 (2010) 55. (Journals)

[8] Jpn. Soc. Mech. Eng. ed.: "JSME Date Handbook: Heat Transfer, (4th Ed.)" (1986) p.321. (in Japanese)

[9] E.B. Shand: "Glass Engineering Handbook, (2nd Ed.)", (Publisher, McGraw-Hill, 1958) p.4 (Books)

[10]M. Yamame, I. Yasui, M. Wada, Y. Kokubu, R. Terai, K. Kondo and S. Ogawa: "Glass Engineering Handbook" (Publisher, Asakura Shoten, 1999) p.441. (Books).

[11]H.D. Hibbitt: Int. J. Num. Meth. Eng,, 11, (2005) 180. (Journals)

[12] R.S. Barsoum: Int. J. Frac., 10 (1974) 603. (Journals)

[13] S. Yoshimura, J.S. Lee, G. Yagawa: Trans. Jpn. Soc. Mech. Eng. A, 61 (1995) 128. (in Japanese).

(Received: July 31, 2009, Accepted: March 1, 2010) 